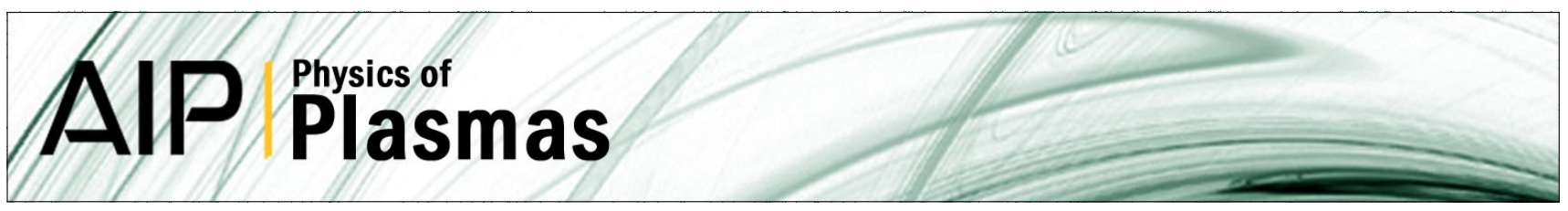

\title{
Asymmetric radiative damping of low shear toroidal Alfvén eigenmodes
}

R. M. Nyqvist and S. E. Sharapov

Citation: Phys. Plasmas 19, 082517 (2012); doi: 10.1063/1.4748292

View online: http://dx.doi.org/10.1063/1.4748292

View Table of Contents: http://pop.aip.org/resource/1/PHPAEN/v19/i8

Published by the American Institute of Physics.

\section{Related Articles}

Enhancement of Ka emission through efficient hot electron generation in carbon nanotubes on intense laser pulse irradiation

J. Appl. Phys. 112, 053301 (2012)

Temporal evolution of dielectric barrier discharge microplasma

Appl. Phys. Lett. 101, 104101 (2012)

Simultaneous imaging electron- and ion-feature Thomson scattering measurements of radiatively heated $\mathrm{Xe}$ Rev. Sci. Instrum. 83, 10E348 (2012)

Spectral emission measurements of lithium on the lithium tokamak experiment

Rev. Sci. Instrum. 83, 10D537 (2012)

Concept to diagnose mix with imaging $\mathrm{x}$-ray Thomson scattering

Rev. Sci. Instrum. 83, 10E534 (2012)

\section{Additional information on Phys. Plasmas}

Journal Homepage: http://pop.aip.org/

Journal Information: http://pop.aip.org/about/about_the_journal

Top downloads: http://pop.aip.org/features/most_downloaded

Information for Authors: http://pop.aip.org/authors

\section{ADVERTISEMENT}

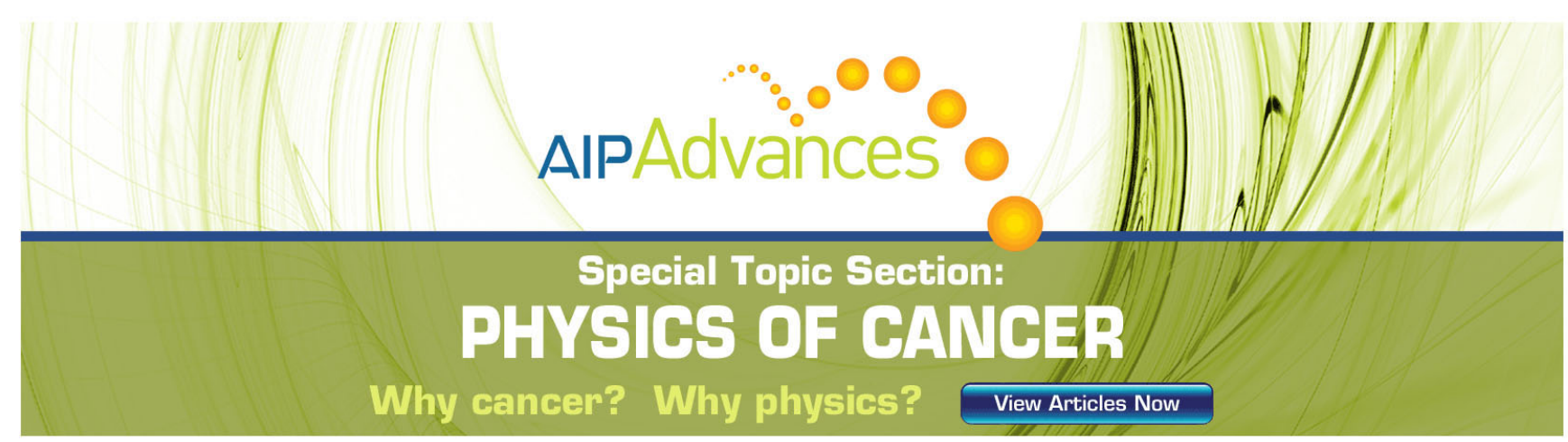




\title{
Asymmetric radiative damping of low shear toroidal Alfvén eigenmodes
}

\author{
R. M. Nyqvist ${ }^{1, a)}$ and S. E. Sharapov ${ }^{2}$ \\ ${ }^{1}$ Department of Earth and Space Sciences, Chalmers University of Technology, SE-412 96 Göteborg, Sweden \\ ${ }^{2}$ EURATOM/CCFE Fusion Association, Culham Science Centre, Abingdon OX14 3DB, United Kingdom
}

(Received 19 June 2012; accepted 13 August 2012; published online 29 August 2012)

\begin{abstract}
Radiative damping of toroidicity-induced Alfvén eigenmodes (TAEs) in tokamaks, caused by coupling to the kinetic Alfvén wave (KAW), is investigated analytically in the limit of low magnetic shear. A significant asymmetry is found between the radiative damping of the odd TAE, whose frequency lies above the central TAE gap frequency $\omega_{0}$, and that of the even TAE, with frequency $\omega<\omega_{0}$. For the even TAE, which consists of a symmetric combination of neighboring poloidal harmonics (and therefore has ballooning-type mode structure), the coupling results in two nonoverlapping, outgoing fluxes of KAWs that propagate radially away from each other and the TAE localization region. In contrast, the odd TAE consists of an antisymmetric combination of neighboring poloidal harmonics, resulting in anti-ballooning mode structure. For this mode, the KAWs initially propagate towards each other and form an interference pattern in the TAE localization region, resulting in a negligibly small escaping flux and a correspondingly low radiative damping rate. As a result of the up/down asymmetry in radiative damping with respect to the mode frequency, the odd TAE may be destabilized by fusion born alpha particles more easily than the usual, even TAE. (C) 2012 American Institute of Physics. [http://dx.doi.org/10.1063/1.4748292]
\end{abstract}

\section{INTRODUCTION}

In burning deuterium-tritium (DT) plasma experiments, such as ITER, ${ }^{1}$ the number and type of kinetic instabilities excited by fusion-born alpha particles may become important factors for alpha particle transport and heating. In particular, toroidal Alfvén eigenmodes $(\mathrm{TAEs})^{2}$ are of major concern since they can be destabilized by super-Alfvénic alpha particles via parallel wave-particle resonances ${ }^{3}$ and subsequently may degrade the alpha particle heating, cause alpha particle losses, and damage the first wall.

The stability of TAEs is determined by a competition between the drive from the alpha particles ${ }^{3}$ and a sum of damping effects caused by the thermal plasma (see, e.g., Ref. 4). Among others, the so called radiative damping, ${ }^{5}$ due to a small but finite coupling between the ideal magnetohydrodynamic (MHD) TAE and the non-ideal kinetic Alfvén wave (KAW), ${ }^{6}$ may become significant. This type of damping is, however, less understood than others, and estimates of the damping rate still have large error bars due to its exponential sensitivity to finite ion Larmor radius (FLR) effects. ${ }^{5,7}$ Moreover, agreement between theory and dedicated experiments has so far not been very convincing. ${ }^{8}$

The aim of this article is to develop analytical theory demonstrating that radiative damping of TAEs with eigenfrequencies at the top end of the toroidicity induced gap is negligible. Such modes are called odd TAEs, as they are formed through the toroidal coupling of poloidal harmonics with opposite signs, and they have anti-ballooning mode structure with maximum amplitude at the high field side of the torus. In contrast, even TAEs, with eigenfrequencies at the bottom of the TAE gap, consist of poloidal harmonics with the same sign, resulting in a ballooning-type mode structure with

\footnotetext{
${ }^{a)}$ Electronic mail: robert.nyqvist@chalmers.se.
}

maximum amplitude on the low field side. Although the antiballooning structure of the odd TAE makes it difficult to excite in present day machines, where super-Alfvénic, trapped ions accelerated by ion cyclotron resonance heating (ICRH) are mostly localized at the low field side of the torus, the weakly damped odd TAE may be destabilized more easily than the usual, even TAE by passing alpha particle resonances in burning plasma experiments.

The very existence of the odd TAE deserves a special comment. Historically, the first theoretical investigation of $\mathrm{TAEs}^{2}$ assumed a toroidal plasma equilibrium satisfying a certain relation between the inverse aspect ratio, $\epsilon \equiv r / R_{0}$, and the magnetic shear, $s \equiv(r / q) d q / d r$, namely

$$
\epsilon \ll s^{2} \text {. }
$$

Here, $R_{0}$ is the major radius at the magnetic axis, $r$ is the minor radius, and $q(r)$ is the safety factor. Under the assumption (1), one discrete eigenvalue was found to exist within the TAE gap (i.e., a single TAE mode per gap). With the same ordering, the effect of finite pressure was shown to suppress the TAE when the normalized pressure gradient

$$
\alpha \equiv-R_{0} q^{2} \frac{d \beta}{d r}
$$

exceeds the critical value ${ }^{9}$

$$
\alpha_{c} \approx \frac{s^{2}}{1+s} .
$$

In these expressions, $\beta=8 \pi p / B_{0}^{2}$ is the ratio between the plasma thermal and magnetic pressures, with $B_{0}$ the magnetic field at the magnetic axis. However, it was found ${ }^{10}$ during the DT campaign on the tokamak fusion test reactor (TFTR) that the equilibria relevant to high pressure TFTR 
plasmas do not satisfy the condition (1) in the region of highest alpha particle pressure and that TAE modes could exist even at normalized plasma pressure gradients well exceeding $\alpha_{c}$ as given by Eq. (3). Subsequent analytical theory ${ }^{11}$ explained the existence of TAEs in high pressure plasmas as the case of $\epsilon \sim s$ equilibria, for which finite aspect ratio effects qualitatively modify the expression (3) to $\alpha_{c} \approx 3 \epsilon+2 s^{2}$. Furthermore, it was found ${ }^{12}$ that two TAEs per gap could exist in TFTR equilibria satisfying $\epsilon \sim s^{2}$ : One with eigenfrequency at the bottom of the gap, consisting of two coupled poloidal harmonics of the same sign (even TAE), and another one with frequency at the top of the gap, consisting of two poloidal harmonics of opposite signs (odd TAE). The even TAE corresponds to the previously found mode, with similar eigenfrequency and $\alpha_{c}$, while the unexpected existence of the odd mode requires that $\epsilon \gtrsim s^{2}$. In fact, the odd mode had previously been noticed in numerical analysis, ${ }^{13}$ but its importance was fully appreciated only when the relevant TFTR equilibria were found to support such modes in real plasmas.

Experimentally, the odd TAE (observed together with the even TAE) was identified in ICRH-heated JET discharges with extended low shear central regions in sawtoothing plasmas. $^{14}$ It was found that the overall temporal evolution of the modes is in agreement with analytical theory,${ }^{11}$ but the proximity of the magnetic axis renders a non-trivial frequency evolution that deviates from the usual TAE scaling $\omega \propto B / \sqrt{n}$.

The important role of low shear TAEs as triggers of monster sawtooth crashes was underlined in experiments on TFTR, ${ }^{15}$ DIII-D, ${ }^{16}$ JET, $,{ }^{17}, 18$ and C-MOD. ${ }^{19}$ Moreover, in addition to the sawtoothing low shear scenarios typical for present-day tokamaks, novel so called hybrid scenarios of enhanced plasma performance were established as good candidates for burning ITER plasmas. ${ }^{20}$ These scenarios also require extended regions of low shear, typically satisfying $s^{2} \leq \epsilon$. Finally, the spherical tokamak approach, ${ }^{21}$ with the aspect ratio close to unity, could enter the very interesting limit $s \ll \epsilon$, where spectra of multiple TAEs are expected to exist within each gap. ${ }^{22}$ Although there has been no unambiguous identification of multiple TAEs on present-day spherical tokamaks, future experiments may change this situation and such modes will have to be investigated in detail.

In this article, we present analytical theory of radiative damping for low shear TAEs due to wave coupling to the KAW. The model is based on the ideal MHD framework, and the TAE/KAW coupling is analyzed by incorporating non-ideal terms due to ion FLR effects and a non-vanishing parallel electric field. The case with both odd and even TAEs is the focus of the study, with the respective eigenfrequencies situated in the proximity of the top and bottom tips of the Alfvén continuum. The paper is organized in the following way: In Sec. II, a set of non-ideal mode equations are derived and analyzed in the inner region surrounding the TAE localization area as well as the outer MHD region. Then, in Sec. III, a matching technique is applied for the analytic solutions in the separate inner and outer regions, which results in dispersion relations for the odd and even TAEs, including the radiative damping. Finally, conclusions are presented and discussed in Sec. V. Note that some of the more lengthy calculations are reproduced in detail in the two appendices, whereas only the corresponding results are given in the main text.

\section{NON-IDEAL MODE EQUATIONS}

In toroidal magnetic confinement devices, the magnetic field topology induces couplings between the poloidal harmonics $\phi_{m}$ of the Fourier expanded, perturbed electrostatic potential

$$
\Phi(r, \theta, \varphi)=\mathrm{e}^{i(n \varphi-\omega t)} \sum_{m} \phi_{m}(r) \mathrm{e}^{-i m \theta} .
$$

Here $(r, \theta, \varphi)$ are the usual toroidal coordinates, $m$ and $n$ are the poloidal and toroidal mode numbers, respectively, and $\omega$ is the mode frequency. In particular, toroidal effects such as the radial dependence of the magnetic field strength and the Shafranov shift of the magnetic axis from the geometric axis couples the dynamics of mainly neighboring poloidal harmonics $\phi_{m}$ and $\phi_{m-1}$. This toroidicity induced coupling is most vigorous at the radial location $r=r_{m}$, where the cylindrical Alfvén continua

$$
\omega_{A}^{2}=k_{\| m}^{2}(r) v_{A}^{2}(r)
$$

for the two neighboring poloidal harmonics cross. In Eq. (5), $v_{A}$ is the Alfvén velocity, and the parallel wave number is given by

$$
k_{\| m}(r)=\frac{n q(r)-m}{q(r) R_{0}} .
$$

Thus, $r_{m}$ is found by setting $k_{\| m-1}=-k_{\| m}$, resulting in the condition $q\left(r_{m}\right)=(m-1 / 2) / n \equiv q_{m}$. In the proximity of $r=r_{m}$, the toroidal coupling induces a gap in the global Alfvén continuum, centered at the frequency

$$
\omega_{0} \equiv k_{\| m}\left(r_{m}\right) v_{A}\left(r_{m}\right)=\frac{v_{A}\left(r_{m}\right)}{2 q_{m} R_{0}},
$$

whose width can be estimated as $\Delta \omega / \omega_{0} \sim \epsilon$. The situation is portrayed qualitatively in Figure 1. As depicted, the coupling also enables the possibility of discrete frequency eigenmodes within the gap, situated close to $r=r_{m}$. These modes are the TAEs discussed in Sec. I. In the remainder of this section, we present and analyze a set of coupled, nonideal mode equations for $\phi_{m}$ and $\phi_{m-1}$, which will ultimately yield TAE dispersion relations, including imaginary parts that represent the radiative damping.

To proceed with formalism, we focus our attention on the case with small inverse aspect ratio, $\epsilon \ll 1$, high poloidal mode number, $m \gg 1$, and low shear, $s \ll 1$. In this limit, each eigenmode is highly localized in the vicinity of $r=r_{m}$ and consists of merely two neighboring poloidal harmonics, $\phi_{m}$ and $\phi_{m-1}$. Assuming circular magnetic flux surfaces, the appropriate set of equations for $\phi_{m}$ and $\phi_{m-1}$ is then given by the following coupled, fourth order differential equations ${ }^{12,23}$ : 


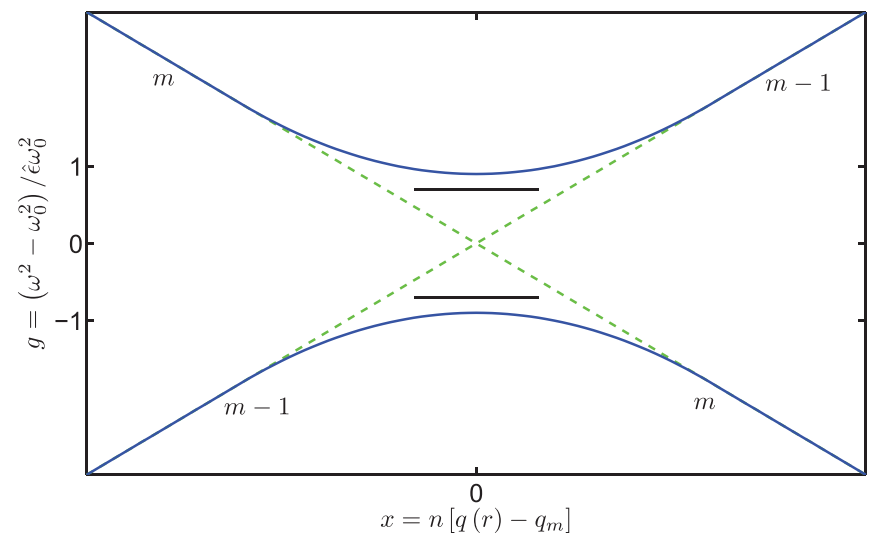

FIG. 1. Qualitative figure of a single toroidicity induced Alfvén gap, with discrete frequency eigenmodes close to the two continuum tips. The green (dashed) lines correspond to the cylindrical continua for the $m$ :th and $m-$ 1:th poloidal harmonics, given by Eq. (5), and the blue (solid) lines constitute the actual, toroidal continuum. Note that sufficiently far from $x=0$, where toroidal effects are unimportant, the two types of curves merge.

$$
\begin{aligned}
& \rho^{2} \frac{d^{4} \phi_{m}}{d r^{4}}+\mathcal{L}_{m} \phi_{m}+\frac{\epsilon+\Delta^{\prime}}{2 q_{m}^{2} R_{0}^{2}} \frac{d^{2} \phi_{m-1}}{d r^{2}}+\frac{m^{2}}{r_{m}^{2}} \frac{\Delta^{\prime}-\alpha}{2 q_{m}^{2} R_{0}^{2}} \phi_{m-1}=0 \\
& \rho^{2} \frac{d^{4} \phi_{m-1}}{d r^{4}}+\mathcal{L}_{m-1} \phi_{m-1}+\frac{\epsilon+\Delta^{\prime}}{2 q_{m}^{2} R_{0}^{2}} \frac{d^{2} \phi_{m}}{d r^{2}}+\frac{m^{2}}{r_{m}^{2}} \frac{\Delta^{\prime}-\alpha}{2 q_{m}^{2} R_{0}^{2}} \phi_{m}=0,
\end{aligned}
$$

with

$$
\mathcal{L}_{m} \equiv \frac{d}{d r}\left[\left(\frac{\omega^{2}}{v_{A}^{2}}-k_{\| m}^{2}(r)\right) \frac{d}{d r}\right]-\frac{m^{2}}{r_{m}^{2}}\left[\frac{\omega^{2}}{v_{A}^{2}}-k_{\| m}^{2}(r)\right] .
$$

In Eqs. (8), the second terms are the cylindrical, low- $\beta$, MHD operators ${ }^{24}$ for $\phi_{m}$ and $\phi_{m-1}$. The first terms, proportional to

$$
\rho^{2} \equiv \frac{\rho_{i}^{2}}{4 q_{m}^{2} R_{0}^{2}}\left[\frac{3}{4}+\tau\right]
$$

accounts for the non-ideal contributions due to first order FLR effects and finite parallel electric field. ${ }^{25,26}$ Here $\rho_{i}$ is the ion Larmor radius and $\tau \equiv T_{e} / T_{i}(1-i \delta)$, with $T_{i}$ and $T_{e}$ the ion and electron temperatures, represents the electron contribution, including a small wave dissipation rate $\delta$. An explicit form for $\delta$ is calculated in Ref. 26, but for our purposes it suffices to know that $0<\delta \ll 1$. The third and fourth terms constitute the toroidicity induced coupling, including the effect of finite plasma pressure. Here, $\Delta^{\prime}$ is the radial derivative of the Shafranov shift, and the normalized pressure gradient, $\alpha$, is given by Eq. (2). Note that $\epsilon, \Delta^{\prime}, \alpha$, and $v_{A}$ are to be evaluated at $r=r_{m}$.

In order to simplify the subsequent analysis, we introduce the normalized frequency $g \equiv\left(\omega^{2}-\omega_{0}^{2}\right) / \hat{\epsilon} \omega_{0}^{2}$, with $\hat{\epsilon} \equiv 2\left(\epsilon+\Delta^{\prime}\right)$, which takes on the values \pm 1 at the upper/ lower tips of the continuum, respectively, and a dimensionless radial variable $x \equiv n\left[q(r)-q_{m}\right]$. The mode equations, Eqs. (8), then transform into

$$
\begin{aligned}
& \bar{\rho}^{2} \frac{d^{4} \phi_{m}}{d x^{4}}+\frac{d}{d x}\left[\left(\frac{\hat{\epsilon}}{4} g+x-x^{2}\right) \frac{d \phi_{m}}{d x}\right] \\
& -\frac{1}{s^{2}}\left[\frac{\hat{\epsilon}}{4} g+x-x^{2}\right] \phi_{m}+\frac{\hat{\epsilon}}{4} \frac{d^{2} \phi_{m-1}}{d x^{2}}+\frac{\Delta^{\prime}-\alpha}{2 s^{2}} \phi_{m-1}=0 \\
& \bar{\rho}^{2} \frac{d^{4} \phi_{m-1}}{d x^{4}}+\frac{d}{d x}\left[\left(\frac{\hat{\epsilon}}{4} g-x-x^{2}\right) \frac{d \phi_{m-1}}{d x}\right] \\
& -\frac{1}{s^{2}}\left[\frac{\hat{\epsilon}}{4} g-x-x^{2}\right] \phi_{m-1}+\frac{\hat{\epsilon}}{4} \frac{d^{2} \phi_{m}}{d x^{2}}+\frac{\Delta^{\prime}-\alpha}{2 s^{2}} \phi_{m}=0
\end{aligned}
$$

where

$$
\bar{\rho}^{2} \equiv \frac{m^{2} s^{2} \rho_{i}^{2}}{4 r_{m}^{2}}\left[\frac{3}{4}+\tau\right]
$$

and $s$ is to be evaluated at the location $r=r_{m}$. Focusing on the situation with two TAEs present in the gap, ${ }^{12}$ we take $\hat{\epsilon}, \Delta^{\prime}$, and $\alpha$ to be $\mathcal{O}\left(s^{2}\right)$, and $\bar{\rho} \ll s^{2}$. With this ordering, Eqs. (11) constitute a boundary layer problem: The $x$-axis separates into an outer region with $x \sim s$ and an inner layer where $x \sim s^{3}$ (this can be shown a priori), characterized by very peaked gradients.

Intuitively, the implications of non-ideal effects on the TAEs can be understood by once again turning to the qualitative configuration in Figure 1: There are two TAEs inside the toroidicity induced gap, shifted slightly off the upper and lower continua. The radiative damping is due to mode conversion of parts of the TAE amplitudes into KAWs that propagate radially away from the TAE localization region close to $x=0$. Noting that the KAW local dispersion relation reads

$$
\omega_{K A W}^{2}=k_{\| m}^{2} v_{A}^{2}\left[1+k_{\perp}^{2} \rho_{i}^{2}(3 / 4+\tau)\right],
$$

so that $\omega_{K A W}^{2}>\omega_{A}^{2}$, one realizes that the KAW with poloidal mode number $m$ can only propagate above the cylindrical continuum for the $m$ :th poloidal harmonic (the curve labeled $m$ in Figure 1). Likewise, the KAW with poloidal mode number $m-1$ can only propagate above the line labeled $m-1$. For the even TAE at the bottom of the gap, the KAW formation thus occurs in the tails of the TAE eigenfunction, resulting in non-overlapping fluxes of outward propagating KAWs. For the odd mode at the top end of the gap, on the other hand, the KAWs form in a joint, intermediate region where they interact and establish an interference pattern. The purpose of this article is to show that the consequent escaping fluxes differ significantly from those of the even TAE, thus leading to an asymmetry in the rates of radiative damping for the odd and even modes.

\section{A. Outer region}

In the outer region where $x \sim s$, we neglect the fourth order derivative FLR terms in Eqs. (11) and introduce the new spatial variable $y=x / s$. Following Ref. 12 , we can then reorganize the terms so that the ordering in $s$ becomes manifest: 


$$
\begin{aligned}
\mathcal{L}_{0} \phi_{m}= & s\left[y \mathcal{L}_{0}+y \frac{d}{d y}-\frac{\hat{\epsilon} g}{4 s^{2}} \frac{d^{2}}{d y^{2}}+\frac{\hat{\epsilon} g}{4 s^{2}}\right] \phi_{m} \\
& -s\left[\frac{\hat{\epsilon}}{4 s^{2}} \frac{d^{2}}{d y^{2}}+\frac{\Delta^{\prime}-\alpha}{2 s^{2}}\right] \phi_{m-1}, \\
\mathcal{L}_{0} \phi_{m-1}= & -s\left[y \mathcal{L}_{0}+y \frac{d}{d y}-\frac{\hat{\epsilon} g}{4 s^{2}} \frac{d^{2}}{d y^{2}}+\frac{\hat{\epsilon} g}{4 s^{2}}\right] \phi_{m-1} \\
& +s\left[\frac{\hat{\epsilon}}{4 s^{2}} \frac{d^{2}}{d y^{2}}+\frac{\Delta^{\prime}-\alpha}{2 s^{2}}\right] \phi_{m},
\end{aligned}
$$

where

$$
\mathcal{L}_{0} \equiv \frac{d}{d y}\left(y \frac{d}{d y}\right)-y .
$$

To lowest order in $s$, the solutions that decay at $|y| \rightarrow \infty$ have even parity, viz.,

$$
\phi_{m}^{0}=-C_{m} K_{0}(|y|),
$$

where $C_{m}$ are constants and $K_{0}$ is the zeroth order modified Bessel function of the second kind. The next order, odd parity corrections to Eq. (16) result in a discontinuity at the origin, with a corresponding jump in $\phi_{m}$,

$$
\Delta \phi_{m} \equiv \lim _{\epsilon \rightarrow 0}\left[\phi_{m}^{1}(\epsilon)-\phi_{m}^{1}(-\epsilon)\right]
$$

that can be calculated as

$$
\Delta \phi_{m}=-s \int_{-\infty}^{\infty} K_{0}(|y|) \mathcal{L}_{0} \phi_{m}^{1} d y .
$$

Substituting Eq. (16) into the right hand sides of Eqs. (14), one finds

$$
\begin{aligned}
\Delta \phi_{m}= & 2 s\left[I_{2}-\frac{\hat{\epsilon} g}{4 s^{2}} I_{3}+\frac{\hat{\epsilon} g}{4 s^{2}} I_{1}\right] C_{m} \\
& -2 s\left[\frac{\hat{\epsilon}}{4 s^{2}} I_{3}+\frac{\Delta^{\prime}-\alpha}{2 s^{2}} I_{1}\right] C_{m-1}, \\
\Delta \phi_{m-1}= & -2 s\left[I_{2}-\frac{\hat{\epsilon} g}{4 s^{2}} I_{3}+\frac{\hat{\epsilon} g}{4 s^{2}} I_{1}\right] C_{m-1} \\
+ & 2 s\left[\frac{\hat{\epsilon}}{4 s^{2}} I_{3}+\frac{\Delta^{\prime}-\alpha}{2 s^{2}} I_{1}\right] C_{m},
\end{aligned}
$$

where

$$
\begin{gathered}
I_{1} \equiv \int_{0}^{\infty} K_{0}^{2}(y) d y=\frac{\pi^{2}}{4}, \\
I_{2} \equiv \int_{0}^{\infty} y K_{0}(y) \frac{d K_{0}}{d y} d y=-\frac{\pi^{2}}{8}, \\
I_{3} \equiv \int_{0}^{\infty} K_{0}(y) \frac{d^{2} K_{0}}{d y^{2}} d y \approx \frac{\pi^{2}}{4} .
\end{gathered}
$$

There is a slight subtlety in the evaluation of $I_{3}$, where an apparent divergence has to be removed. The reader is referred to Ref. 12, where these technicalities are discussed in more detail. With Eqs. (20), the jumps become

$$
\begin{gathered}
\Delta \phi_{m}=-\frac{\pi^{2} s}{4}\left[C_{m}+\frac{\tilde{\epsilon}-\alpha}{s^{2}} C_{m-1}\right], \\
\Delta \phi_{m-1}=\frac{\pi^{2} s}{4}\left[C_{m-1}+\frac{\tilde{\epsilon}-\alpha}{s^{2}} C_{m}\right],
\end{gathered}
$$

where $\tilde{\epsilon} \equiv \epsilon+2 \Delta^{\prime}$.

\section{B. Inner layer}

In the inner layer, the solutions to Eqs. (11) are highly peaked, so only the highest order derivates contribute. Neglecting lower order derivatives and approximating $x(1 \pm x) \approx x$, the inner layer mode equations can be written as $^{23}$

$$
\begin{gathered}
\lambda^{2} \frac{d^{4} \phi_{m}}{d z^{4}}+\frac{d}{d z}\left[(g+z) \frac{d \phi_{m}}{d z}\right]+\frac{d^{2} \phi_{m-1}}{d z^{2}}=0, \\
\lambda^{2} \frac{d^{4} \phi_{m-1}}{d z^{4}}+\frac{d}{d z}\left[(g-z) \frac{d \phi_{m-1}}{d z}\right]+\frac{d^{2} \phi_{m}}{d z^{2}}=0,
\end{gathered}
$$

where $z \equiv 4 x / \hat{\epsilon}$ and the FLR terms are to be regarded as small perturbations, with

$$
\lambda^{2} \equiv(4 / \hat{\epsilon})^{3} \bar{\rho}^{2} \ll 1 .
$$

Setting $U \equiv d \phi_{m} / d z$ and $V \equiv d \phi_{m-1} / d z$ and integrating once yields

$$
\begin{gathered}
\lambda^{2} \frac{d^{2} U}{d z^{2}}+(g+z) U+V=C_{m}, \\
\lambda^{2} \frac{d^{2} V}{d z^{2}}+(g-z) V+U=-C_{m-1} .
\end{gathered}
$$

Here, the integration constants have been chosen so that the large $z$ asymptotes of $U$ and $V$ match onto the $y \ll 1$, lowest order solutions in the outer region, i.e., Eq. (16). ${ }^{23}$

The form of the solutions to Eqs. (24) can be seen by first neglecting the small FLR terms. Equations (24) then become algebraic in $U$ and $V$, and the corresponding solutions for $\phi_{m}$ and $\phi_{m-1}$ are found to consist of two pieces ${ }^{23}$ : An even part that matches onto Eq. (16) when $z \gg 1$, and an odd part that produces jumps in $\phi_{m}$ and $\phi_{m-1}$ across the inner layer. To obtain dispersion relations in the absence of non-ideal effects, these jumps must then match onto the jumps at the origin of the outer region solutions, i.e., Eqs. (21). The presence of the small FLR terms in Eqs. (24), however, changes only the form of the jumps slightly. Following the method developed in Refs. 26 and 27, we choose to work with the Fourier space versions of Eqs. (24). The jumps in $\phi_{m}$ and $\phi_{m-1}$ are then given by (cf., Appendix A) 


$$
\Delta \phi_{m}=\pi\left[\alpha C_{m}+\beta C_{m-1}\right], \quad \Delta \phi_{m-1}=-\pi\left[\alpha C_{m-1}+\beta C_{m}\right],
$$

where

$$
\alpha=-i \frac{\mu^{2}+1}{\mu^{2}-1}, \quad \beta=i \frac{2 \mu}{\mu^{2}-1},
$$

and

$$
\mu=-\frac{1}{g+i S}
$$

Here the parameter $S$ is most easily obtained as

$$
S=\lim _{k \rightarrow 0} \frac{1}{u} \frac{d u}{d k}
$$

where $u$ is the solution to the Fourier space boundary value problem

$$
\frac{d^{2} u}{d k^{2}}=Q u
$$

with

$$
Q \equiv 1-\left(g-\lambda^{2} k^{2}\right)^{2}-2 i \lambda^{2} k
$$

given that $u \rightarrow 0$ as $k \rightarrow \infty$.

\section{DISPERSION RELATIONS}

The dispersion relation is obtained by equating the jumps across the inner layer, given by Eq. (25), with the outer region jumps at the origin (Eqs. (21)). After some algebra, the resulting expressions take the form

$$
\begin{aligned}
& \left(C_{m}-C_{m-1}\right)\left[(\alpha-\beta)-\frac{\pi s}{4} A_{-}\right]=0, \\
& \left(C_{m}+C_{m-1}\right)\left[(\alpha+\beta)+\frac{\pi s}{4} A_{+}\right]=0,
\end{aligned}
$$

where

$$
A_{ \pm} \equiv \frac{\tilde{\epsilon}-\alpha}{s^{2}} \pm 1
$$

Equations (31) allow for the two distinct solutions

$$
C_{m}= \pm C_{m-1}
$$

with

$$
\alpha \pm \beta=\mp \frac{\pi s}{4} A_{ \pm} .
$$

Using the expressions (26) and (27), we also have

$$
\alpha \pm \beta=i \frac{1 \mp \mu}{1 \pm \mu}=-i \frac{1 \pm g \pm i S}{1 \mp g \mp i S},
$$

where the parameter $S$ is calculated in Appendix B.

\section{A. Even mode, $C_{m}=C_{m-1}$}

This is the usual TAE mode, consisting of a symmetric combination of the poloidal harmonics $\phi_{m}$ and $\phi_{m-1}$. The eigenfrequency lies just above the tip of the lower Alfvén continuum, with $0<1+g \ll 1$, and the appropriate form of $S$ is given by (cf., Appendix B1)

$$
S=-\sqrt{1-g^{2}}\left[1+i \mathrm{e}^{-\pi a}\right]
$$

where

$$
a=\frac{1-g^{2}}{\sqrt{8} \lambda} .
$$

In the limit $a \gg 1$, the condition (33b) can be written as

$$
\sqrt{\frac{1+g}{1-g}}\left[1+i \mathrm{e}^{-\pi a}\right]=\frac{\pi s}{4} A_{+},
$$

which requires that $A_{+}>0$, i.e.,

$$
\alpha<\tilde{\epsilon}+s^{2}=\epsilon+2 \Delta^{\prime}+s^{2} .
$$

Note that Eq. (38) corresponds to the value for $\alpha_{c}$ derived in Ref. 12 for the even mode. The dispersion relation (37) may be iteratively solved in the large- $a$ limit to yield

$$
g=-\left[1-\frac{\pi^{2} s^{2}}{8} A_{+}^{2}+i \frac{\pi^{2} s^{2}}{4} A_{+}^{2} \exp \left[-\frac{\pi^{3} s^{2}}{8 \sqrt{2} \lambda} A_{+}^{2}\right]\right],
$$

so that the radiative damping rate becomes

$$
\frac{\gamma}{\omega}=-\hat{\epsilon} \frac{\pi^{2} s^{2}}{8}\left[\frac{\tilde{\epsilon}-\alpha}{s^{2}}+1\right]^{2} \exp \left[-\frac{\pi^{3} s^{2}}{8 \sqrt{2} \lambda}\left[\frac{\tilde{\epsilon}-\alpha}{s^{2}}+1\right]^{2}\right]
$$

\section{B. Odd mode, $C_{m}=-C_{m-1}$}

This case corresponds to an antisymmetric combination of the poloidal harmonics $\phi_{m}$ and $\phi_{m-1}$, and its eigenfrequency resides close to the upper tip of the Alfvén continuum, where $0<1-g \ll 1$. The appropriate form for $S$ is (cf., Appendix B2)

$$
S=-\sqrt{1-g^{2}}[1+i 4 \exp (-4 \sqrt{2} / 3 \lambda)]
$$

and the matching condition (33b) becomes

$$
\sqrt{\frac{1-g}{1+g}}[1-i 4 \exp (-4 \sqrt{2} / 3 \lambda)]=\frac{\pi s}{4} A_{-},
$$

which can be satisfied only if $A_{-}>0$. Hence, existence of the odd mode requires that

$$
\alpha<\tilde{\epsilon}-s^{2} \approx \epsilon+2 \Delta^{\prime}-s^{2}
$$

which puts a more restrictive limit on the pressure gradient than for the even mode (cf., Eq. (38) and Ref. 12). Moreover, for a centrally peaked, monotonic pressure profile, $\Delta^{\prime}$ can be 
evaluated as a function of $\epsilon$ and $s .{ }^{11}$ Then, Eq. (43) implies that the shear and inverse aspect ratio must satisfy the relation $3 \epsilon>2 s^{2}$ for the odd mode to exist.

When Eq. (43) is satisfied, the dispersion relation (42) may be solved iteratively to yield the normalized frequency

$$
g=1-\frac{\pi^{2} s^{2}}{8}-i \pi^{2} s^{2} A_{-}^{2} \exp (-4 \sqrt{2} / 3 \lambda) .
$$

The radiative damping rate for the odd mode is then

$$
\frac{\gamma}{\omega}=-\hat{\epsilon} \frac{\pi^{2} s^{2}}{2}\left[\frac{\tilde{\epsilon}-\alpha}{s^{2}}-1\right]^{2} \exp (-4 \sqrt{2} / 3 \lambda) .
$$

\section{CONCLUSIONS}

In this article, we have presented analytical theory describing radiative damping of low shear TAEs. The damping is due to so called wave tunneling: Loosely speaking, a part of the TAE amplitude is converted into non-ideal waves that propagate radially away from the eigenmode. The resulting expressions (40) and (45) reveal that the rate of radiative damping of the odd TAE is always exponentially small, as opposed to the even TAE, where the effect becomes significant as $s^{2} \rightarrow \lambda$. Since the even and odd modes have nearly the same frequency and thus are expected to experience approximately the same fast particle drive, this asymmetry suggests that the odd mode may be more easily excited than the even mode.

The odd TAE is rarely observed in experiments,,${ }^{14}$ however, which is partly due to the more restrictive constraint (43) on the odd mode pressure gradient. As previously explained, another important difference between the odd and even mode can be found in the structure of their respective eigenmodes, which are formed from symmetric/antisymmetric combinations of neighboring poloidal harmonics, respectively. The even TAE is therefore a ballooning-type mode, so it resides on the outboard side where it is accessible to both passing and trapped fast particles. The odd mode, on the other hand, is anti-ballooning. It is therefore situated on the inboard side, where it is much more prone to interact with passing fast particles than trapped ones. On JET, e.g., super-Alfvénic fast particles are created by ICRH, and the odd TAE has only been observed with the ICRH resonance layer shifted to the high field side. ${ }^{14}$ In burning ITER plasmas, however, the situation is expected to be the opposite, with enough passing alpha particles available to destabilize the weakly damped odd TAE.

\section{ACKNOWLEDGMENTS}

The authors gratefully acknowledge helpful discussions with H. L. Berk, B. N. Breizman, and M. Lisak. This work was funded jointly by the Swedish Research Council, the RCUK Energy Programme under grant EP/I501045, and the European Communities under the contract of Association between EURATOM and CCFE. The views and opinions expressed herein do not necessarily reflect those of the European Commission.

\section{APPENDIX A: CALCULATION OF INNER LAYER JUMPS IN FOURIER SPACE}

Note that the calculations presented in this section follow exactly the derivation given in Ref. 26. It is repeated here for reasons of completeness only. We start by Fourier transforming the inner layer mode equations (24). Denoting the respective Fourier transforms of $U(z)$ and $V(z)$ with $u(k)$ and $v(k)$, we then have

$$
\begin{gathered}
\mathcal{L}_{k} u+v=2 \pi C_{m} \delta(k), \\
\mathcal{L}_{-k} v+u=-2 \pi C_{m-1} \delta(k),
\end{gathered}
$$

where

$$
\mathcal{L}_{k} \equiv i \frac{d}{d k}+g-\lambda^{2} k^{2},
$$

which are to be solved given that $u, v \rightarrow 0$ as $k \rightarrow \infty$. The presence of the $\delta$-functions on the right hand sides of Eqs. (A1) leads to discontinuities in $u$ and $v$ at the origin, given by the jump conditions

$$
\begin{gathered}
u\left(0^{+}\right)-u\left(0^{-}\right)=-2 \pi i C_{m}, \\
v\left(0^{+}\right)-v\left(0^{-}\right)=-2 \pi i C_{m-1} .
\end{gathered}
$$

On the other hand, away from $k=0$, the two coupled Fourier space equations can be combined into second order, uncoupled equations,

$$
\left[\mathcal{L}_{\mp k} \mathcal{L}_{ \pm k}-1\right]\left[\begin{array}{c}
u \\
v
\end{array}\right]=0
$$

where the upper sign applies for $u$ and the lower for $v$. From Eq. (A4), it can be seen that $u$ and $v$ obey the following symmetry rules for $k>0$ :

$$
u(k)=\sigma v(-k), \quad v(k)=\sigma u(-k),
$$

with $\sigma$ constant. This may be utilized in the calculation of the jumps in $\phi_{m}$ and $\phi_{m-1}$ across the inner layer, yielding

$$
\begin{aligned}
\Delta \phi_{m} & =\phi_{m}(z \rightarrow \infty)-\phi_{m}(z \rightarrow-\infty) \\
& =\int_{-\infty}^{\infty} U d z=\frac{u\left(0^{+}\right)+u\left(0^{-}\right)}{2} \\
& =\pi\left[\alpha C_{m}+\beta C_{m-1}\right],
\end{aligned}
$$

and, similarly,

$$
\Delta \phi_{m-1}=-\pi\left[\alpha C_{m-1}+\beta C_{m}\right],
$$

where

$$
\alpha \equiv-i \frac{\mu^{2}+1}{\mu^{2}-1}, \quad \beta \equiv \frac{2 i \mu}{\mu^{2}-1},
$$

and

$$
\mu \equiv \frac{u\left(0^{+}\right)}{v\left(0^{+}\right)}=\frac{v\left(0^{-}\right)}{u\left(0^{-}\right)} .
$$


An alternative expression for $\mu$ is provided by defining the parameter

$$
S \equiv \lim _{k \rightarrow 0} \frac{1}{u(k)} \frac{d u}{d k}
$$

For $k>0$, we then have

$$
S=\lim _{k \rightarrow 0^{+}} \frac{i\left(g-\lambda^{2} k^{2}\right) u+i v}{u}=i\left[g+\frac{1}{\mu}\right],
$$

so that

$$
\mu=\frac{-1}{g+i S}
$$

In prospect, the jumps across the inner layer can be readily calculated once the boundary value problem

$$
\left[\mathcal{L}_{-k} \mathcal{L}_{k}-1\right] u=0,
$$

or

$$
\frac{d^{2} u}{d k^{2}}=\left[1-\left(g-\lambda^{2} k^{2}\right)^{2}-2 i \lambda^{2} k\right] u,
$$

has been solved for $k>0$, given that $u \rightarrow 0$ as $k \rightarrow \infty$.

\section{APPENDIX B: CALCULATION OF $S$}

In order to evaluate the parameter $S$, we first need to solve Eq. (29) for $u$. Due to the small and negative imaginary part of $\lambda^{2}$, the large- $k$ WKB solution to Eq. (29) must be that of an outgoing wave, viz.,

$$
u(k) \sim \exp \left(-i \lambda^{2} k^{3} / 3\right), \quad k \rightarrow \infty
$$

Note that for a fixed mode frequency, the local KAW dispersion relation (13) predicts that $k_{\|}^{2}$ will decrease as this nonideal wave propagates outward in $k$-space (and $k_{\perp}^{2}$ increases). The asymptote (B1) therefore corresponds to a radially outgoing KAW in real space (as measured from the TAE localization region at $x=0$, cf., Figure 1), and its fractional amplitude will determine the rate of radiative damping.

On the other hand, in the limit of moderate $k$ (to be more precise, for $k \lesssim \lambda^{-1}$ ), we may neglect the imaginary part of $Q$, giving

$$
Q \approx 1-\left(g-\lambda^{2} k^{2}\right)^{2}
$$

Equation (29) with (B2) can then be cast in the form of an equivalent Schrödinger-type eigenvalue problem

$$
\frac{d^{2} u}{d k^{2}}+[E-V(k)] u=0
$$

with $E \equiv-\left(1-g^{2}\right)<0$ for eigenfrequencies in the gap, and

$$
V(k) \equiv 2 g \lambda^{2} k^{2}-\lambda^{4} k^{4}
$$

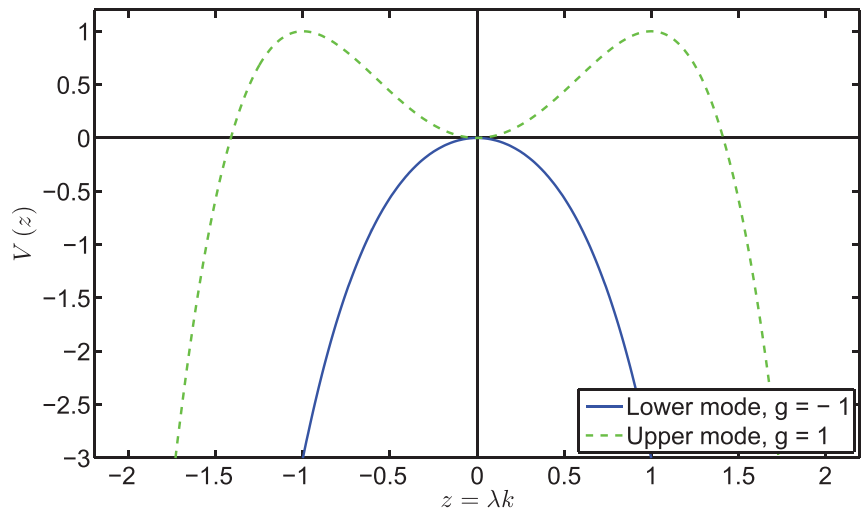

FIG. 2. Effective potential $V(k)$ for the Fourier space Schrödinger problem (B3) when $g= \pm 1$. The radiative damping depends on how large fractions of the TAE amplitudes that tunnel through these potential barriers.

The effective potential $V$ is plotted in Figure 2 for the reference values $g= \pm 1$. The problem at hand then consists of investigating what fraction of $u$ tunnels through the effective potential barrier from $k=0$ all the way to the real turning points

$$
k_{ \pm}= \pm \frac{\sqrt{1+g}}{\lambda}
$$

where $Q=0$, and eventually matches onto the solution (B1) as it propagates towards larger $k$.

It is however obvious from Figure 2 that the thickness of the upper mode potential barrier by far exceeds that of the lower mode. Taking into account the well-known exponential decay of wave functions across potential barriers, it is therefore qualitatively easy to see that the tunnelling through the thick barrier for the upper mode is exponentially smaller than the tunnelling through the much thinner lower mode barrier. Correspondingly, the radiative damping of the upper mode due to the tunnelling is exponentially weaker than the radiative damping of the lower mode. However, an accurate assessment must also include the pre-exponent factors and is therefore quite involved. In the following two sections, such an analysis is carried out by means of a rather tedious asymptotic matching technique. From Ref. 12, we expect the eigenfrequencies to lie close to the continuum tips, with $1 \pm g \sim s^{2}$. We therefore adopt the ordering $0<\lambda \ll$ $1 \pm g \ll 1$ throughout this section.

\section{Lower mode, $g \geq-1$}

For a mode with eigenfrequency just above the tip of the lower continuum, the appropriate ordering is $0<\lambda \ll 1$ $+g \ll 1$. For $k \leq k_{+}$, we can then neglect the term $\lambda^{4} k^{4}$ in $Q$ as compared to $2 g \lambda^{2} k^{2}$ and $1-g^{2}$, yielding the parabolic cylinder equation

$$
\frac{d^{2} u}{d y^{2}}+\left[\frac{y^{2}}{4}-a\right] u=0
$$

where

$$
y \equiv \delta k, \quad \delta \equiv\left(8|g| \lambda^{2}\right)^{1 / 4} \approx\left(8 \lambda^{2}\right)^{1 / 4},
$$


and

$$
a \equiv \frac{1-g^{2}}{\delta^{2}} \gg 1
$$

The solution to Eq. (B6) that matches onto Eq. (B1) is given by $^{28}$

$$
u(y)=\text { Const. }[W(a, y)+i \eta W(a,-y)],
$$

where $W$ is a Whittaker function, and

$$
\eta=\sqrt{1+\mathrm{e}^{2 \pi a}}-\mathrm{e}^{\pi a} \approx \frac{\mathrm{e}^{-\pi a}}{2} .
$$

Taylor expanding around $y=0$ and transforming back to $k$ gives

$$
u(k) \approx \text { Const. }\left[1+k \delta \frac{W^{\prime}(a, 0)}{W(a, 0)} \frac{1+i \eta}{1-i \eta}\right], \quad k \rightarrow 0,
$$

where one can show that, for large $a$,

$$
\frac{W^{\prime}(a, 0)}{W(a, 0)} \approx-\sqrt{a} \text {. }
$$

Hence,

$$
u(k) \approx \text { Const. }\left[1-k \sqrt{1-g^{2}}\left[1+i \mathrm{e}^{-\pi a}\right]\right],
$$

so that

$$
S=-\sqrt{1-g^{2}}\left[1+i \mathrm{e}^{-\pi a}\right] .
$$

\section{Upper mode, $g \leqq 1$}

When the mode eigenfrequency lies just below the upper continuum tip, the terms are ordered as $0<\lambda \ll 1-g \ll 1$. The turning points then become approximately $k_{ \pm} \approx \pm \sqrt{2} / \lambda$. Due to the larger value of $k_{+}$, the $\lambda^{4} k^{4}$-term may not be altogether neglected (as in the case of the lower mode), and the corresponding matching procedure for the upper mode turns out to be more strenuous than for the lower mode: In total, one has to find, and match asymptotically, solutions in 3 different regions for $0 \leq k \leq \lambda^{-1}$, given the large- $k$ form (B1).

Region $1, k \ll \lambda^{-1}$ : As for the lover mode, the $\lambda^{4} k^{4}$ term may be neglected in this region, resulting in

$$
Q \approx \delta^{2}\left[\frac{y^{2}}{4}+a\right]
$$

where $y$ and $a$ are defined in Eqs. (B7) and (B8). The general solution to the corresponding form of Eq. (29), i.e.,

$$
\frac{d^{2} u}{d y^{2}}-\left[\frac{y^{2}}{4}+a\right] u=0,
$$

is then given in terms of parabolic cylinder functions ${ }^{28}$

$$
u(y)=A_{1} U(a, y)+B_{1} V(a, y),
$$

with $A_{1}$ and $B_{1}$ constants. As $y \rightarrow 0, U$ and $V$ can be expanded to yield

$u(k)=\gamma\left\{1-k \delta \sqrt{2} \tan \left[\pi\left(\frac{1}{4}+\frac{a}{2}\right)\right] \frac{\Gamma\left(\frac{3}{4}-\frac{a}{2}\right)}{\Gamma\left(\frac{1}{4}-\frac{a}{2}\right)} \frac{1-\frac{B_{1}}{A_{1}} \frac{\cot \left[\pi\left(\frac{1}{4}+\frac{a}{2}\right)\right]}{\Gamma\left(\frac{1}{2}-a\right)}}{1+\frac{B_{1}}{A_{1}} \frac{\tan \left[\pi\left(\frac{1}{4}+\frac{a}{2}\right)\right]}{\Gamma\left(\frac{1}{2}-a\right)}}\right\}$,

where $\gamma$ is a new constant and we have transformed back to $k$. Note that the expression (B18) immediately gives $S$ as

$$
S=-\delta \sqrt{2} \tan \left[\pi\left(\frac{1}{4}+\frac{a}{2}\right)\right] \frac{\Gamma\left(\frac{3}{4}-\frac{a}{2}\right)}{\Gamma\left(\frac{1}{4}-\frac{a}{2}\right)} \frac{1-\frac{B_{1}}{A_{1}} \frac{\cot \left[\pi\left(\frac{1}{4}+\frac{a}{2}\right)\right]}{\Gamma\left(\frac{1}{2}-a\right)}}{1+\frac{B_{1}}{A_{1}} \frac{\tan \left[\pi\left(\frac{1}{4}+\frac{a}{2}\right)\right]}{\Gamma\left(\frac{1}{2}-a\right)}} .
$$

The remaining task is then to derive an expression for the ratio $B_{1} / A_{1}$, which is in fact uniquely determined by the large$k$ solution (B1). However, (B17) does not match directly onto (B1). It turns out that we need to consider two more, intermediate, regions, and for this purpose we need the asymptotic form of Eq. (B17)

$$
u(y)=A_{1} \mathrm{e}^{-y^{2} / 4} y^{-a-1 / 2}\left[1+\frac{B_{1}}{A_{1}} \sqrt{\frac{2}{\pi}} \mathrm{e}^{y^{2} / 2} y^{2 a}\right],
$$

valid for $y \gg 1$.

Region 2, $0<k<k_{+}$: Here, the solution is taken as the WKB form

$$
u(k)=\frac{A_{2}}{Q^{1 / 4}} \exp \left[\int_{0}^{k} \sqrt{Q} d k\right]+\frac{B_{2}}{Q^{1 / 4}} \exp \left[-\int_{0}^{k} \sqrt{Q} d k\right],
$$

where $A_{2}$ and $B_{2}$ are constants. To proceed, we separate the analysis into two parts: First, we match Eq. (B21) onto (B20) for moderately small values of $k, \lambda^{-1} \sqrt{1-g} \ll k \ll \lambda^{-1}$. As for the calculations in region 1 , we may then neglect the $\lambda^{4} k^{4}$-term in $Q$, resulting in

$$
\begin{aligned}
\int_{0}^{k} \sqrt{Q} d k= & \frac{1}{4}\left[y \sqrt{y^{2}+4 a}\right. \\
& \left.+4 a\left(\ln \left|y+\sqrt{y^{2}+4 a}\right|-\ln 2 \sqrt{a}\right)\right] .
\end{aligned}
$$

With the ordering $k \gg \lambda^{-1} \sqrt{1-g}$ we have $y \gg 2 \sqrt{a}$, so that

$$
\int_{0}^{k} \sqrt{Q} d k \approx \frac{y^{2}}{4}+a \ln y-a \ln \sqrt{a} .
$$

The WKB solution (B21) can then be cast into a form similar to Eq. (B20),

$$
u(y)=B_{2} \sqrt{\frac{2}{\delta}} a^{a / 2} \mathrm{e}^{-y^{2} / 4} y^{-a-1 / 2}\left[1+\frac{A_{2}}{B_{2}} a^{-a} \mathrm{e}^{y^{2} / 2} y^{2 a}\right],
$$


where the matching conditions can be immediately read off by comparing with Eq. (B20):

$$
\begin{aligned}
& \frac{B_{1}}{A_{1}}=\frac{A_{2}}{B_{2}} \sqrt{\frac{\pi}{2}} a^{-a}, \\
& A_{1}=B_{2} \sqrt{\frac{2}{\delta}} a^{a / 2} .
\end{aligned}
$$

Second, close to the turning point we write

$$
\int_{0}^{k} \sqrt{Q} d k=\int_{0}^{k_{+}} \sqrt{Q} d k-\int_{k}^{k_{+}} \sqrt{Q} d k
$$

Now, $\lambda^{4} k^{4}$ cannot be neglected in $Q$, and we get

$$
\begin{aligned}
I & \equiv \int_{0}^{k_{+}} \sqrt{Q} d k \\
& =\frac{1}{\lambda} \int_{0}^{\sqrt{1+g}} \sqrt{1-\left(z^{2}-g\right)^{2}} d z \\
& =\frac{\sqrt{2}}{3 \lambda}\left[(1-g) K\left(\sqrt{\frac{1+g}{2}}\right)+2 g E\left(\sqrt{\frac{1+g}{2}}\right)\right] \\
& \approx \frac{\sqrt{2}}{3 \lambda}\left[\frac{1-g}{2} \ln \frac{32}{1+g}+2 g\right] \approx \frac{2 \sqrt{2} g}{3 \lambda},
\end{aligned}
$$

where $K$ and $E$ are the complete elliptic integrals of the first and second kind, respectively, and we have expanded $K$ and $E$ for small $1-g$ on the final line. To evaluate the second integral in Eq. (B26), we set

$$
k=k_{+}-\frac{z}{\nu}, \quad \nu \equiv\left(4 \lambda^{2} k_{+}\right)^{1 / 3} .
$$

For $z \ll\left(2 / \lambda^{2}\right)^{1 / 3}$, we then have

$$
Q \approx \nu^{2} z
$$

so that

$$
\int_{k}^{k_{+}} \sqrt{Q} d k \approx \frac{2}{3} z^{3 / 2}
$$

Hence, the WKB solution for $k \leqq k_{+}$becomes

$$
u(z)=\frac{A_{2} \mathrm{e}^{I}}{\sqrt{\nu} z^{1 / 4}}\left[\exp \left(\frac{2}{3} z^{3 / 2}\right)+\frac{B_{2}}{A_{2}} \mathrm{e}^{-2 I} \exp \left(-\frac{2}{3} z^{3 / 2}\right)\right]
$$

Region 3, $k \sim k_{+}$: Here, Eq. (29) can be written as

$$
\frac{d^{2} u}{d z^{2}}=z u,
$$

where $z$ is defined in Eq. (B28). The general solution to Eq. (B32) is given in terms of the Airy functions

$$
u(z)=A_{3} \operatorname{Ai}(z)+B_{3} \operatorname{Bi}(z)
$$

with $A_{3}$ and $B_{3}$ constants. For large, positive $z, \mathrm{Ai}$ and $\mathrm{Bi}$ may be expanded to give

$$
u(z)=\frac{B_{3}}{\sqrt{\pi} z^{1 / 4}}\left[\exp \left(\frac{2}{3} z^{3 / 2}\right)+\frac{A_{3}}{2 B_{3}} \exp \left(-\frac{2}{3} z^{3 / 2}\right)\right],
$$

which matches onto Eq. (B31) if

$$
\begin{gathered}
\frac{A_{3}}{2 B_{3}}=\frac{B_{2}}{A_{2}} \mathrm{e}^{-2 I}, \\
\frac{B_{3}}{\sqrt{\pi}}=\frac{A_{2} \mathrm{e}^{I}}{\sqrt{\nu}} .
\end{gathered}
$$

For large negative $z$, on the other hand,

$$
u(z) \sim A_{3} \sin \left[\frac{2}{3}(-z)^{3 / 2}+\frac{\pi}{4}\right]+B_{3} \cos \left[\frac{2}{3}(-z)^{3 / 2}+\frac{\pi}{4}\right]
$$

which matches onto the negative phase component (B1) only if $A_{3}=-i B_{3}$. Hence,

$$
\frac{A_{2}}{B_{2}}=\frac{2 B_{3}}{A_{3}} \mathrm{e}^{-2 I}=2 i \mathrm{e}^{-2 I},
$$

so that

$$
\frac{B_{1}}{A_{1}}=\sqrt{2 \pi} i \mathrm{e}^{-2 I} a^{-a} \approx \sqrt{2 \pi} i \mathrm{e}^{-\frac{4 \sqrt{2}}{3 i}} a^{-a}
$$

Now, Eq. (B19) with (B38) defines the parameter $S$ for the upper mode. That is,

$$
S=-\sqrt{2} \delta \tan \xi \frac{\Gamma\left(\frac{3}{4}-\frac{a}{2}\right)}{\Gamma\left(\frac{1}{4}-\frac{a}{2}\right)} \frac{1-i \Delta \cot \xi}{1+i \Delta \tan \xi},
$$

where

$$
\xi \equiv \pi\left(\frac{1}{4}+\frac{a}{2}\right), \quad \Delta \equiv \frac{\sqrt{2 \pi} a^{-a}}{\Gamma\left(\frac{1}{2}-a\right)} \mathrm{e}^{-\frac{4 \sqrt{2}}{3 \lambda}}
$$

In the limit $a \gg 1$, however, it is possible to simplify these expressions somewhat. Using the reflection formula for the gamma function together with Stirling's formula yields ${ }^{28}$

$$
\tan \xi \frac{\Gamma\left(\frac{3}{4}-\frac{a}{2}\right)}{\Gamma\left(\frac{1}{4}-\frac{a}{2}\right)}=\frac{\Gamma\left(\frac{3}{4}+\frac{a}{2}\right)}{\Gamma\left(\frac{1}{4}+\frac{a}{2}\right)} \approx \sqrt{\frac{a}{2}},
$$

and

$$
\Delta \cot \xi \approx 4 \mathrm{e}^{-\frac{4 \sqrt{2}}{3 \lambda}} \cos ^{2} \xi, \quad \Delta \tan \xi \approx 4 \mathrm{e}^{-\frac{4 \sqrt{2}}{3 \lambda}} \sin ^{2} \xi .
$$

Hence, for $a \gg 1$,

$$
\begin{aligned}
S & \approx-\delta \sqrt{a}[1-i \Delta(\cot \xi+\tan \xi)] \\
& \approx-\sqrt{1-g^{2}}[1-i 4 \exp (-4 \sqrt{2} / 3 \lambda)] .
\end{aligned}
$$


${ }^{1}$ A. Fasoli, C. Gormenzano, H. L. Berk, B. N. Breizman, S. Briguglio, D. S. Darrow, N. N. Gorelenkov, W. W. Heidbrink, A. Juan, S. V. Konovalov, R. Nazikian, J. M. Noterdaeme, S. E. Sharapov, D. Testa, K. Tobita, Y. Todo, G. Vlad, and F. Zonca, Nucl. Fusion 47, S264 (2007).

${ }^{2}$ C. Z. Cheng, L. Chen, and M. S. Chance, Ann. Phys. 161, 21 (1985).

${ }^{3}$ G. Y. Fu and J. W. Van Dam, Phys. Fluids B 1, 1949 (1989).

${ }^{4}$ J. W. Connor, R. O. Dendy, R. J. Hastie, D. Borba, G. T. A. Huysmans, W. Kerner, and S. E. Sharapov, in 21st EPS Conference on Controlled Fusion and Plasma Physics, Montpellier, 27 June-1 July 1994, Vol. 18B, Part II, p. 616.

${ }^{5}$ R. Mett and S. Mahajan, Phys. Fluids B 4, 2885 (1992).

${ }^{6}$ A. Hasegawa and L. Chen, Phys. Fluids 19, 1924 (1976).

${ }^{7}$ D. Borba, H. L. Berk, B. N. Breizman, A. Fasoli, F. Nabais, S. D. Pinches, S. E. Sharapov, and D. Testa, Nucl. Fusion 42, 1029 (2002).

${ }^{8}$ D. Testa, G. Y. Fu, A. Jaun, O. Sauter, and JET-EFDA Contributors, Nucl. Fusion 43, 479 (2003).

${ }^{9}$ G. Y. Fu and C. Z. Cheng, Phys. Fluids B 2, 985 (1990).

${ }^{10}$ G. Y. Fu, C. Z. Cheng, R. Budny, Z. Chang, D. S. Darrow, E. Fredrickson, E. Mazzucato, R. Nazikian, and S. Zweben, Phys. Rev. Lett. 75, 2336 (1995).

${ }^{11}$ G. Y. Fu, Phys. Plasmas 2(4), 1029 (1995).

${ }^{12}$ H. L. Berk, J. W. Van Dam, D. Borba, J. Candy, T. A. Huysmans, and S. E. Sharapov, Phys. Plasmas 2(9), 3401 (1995).

${ }^{13}$ S. Poedts, W. Kerner, J. P. Goedbloed, B. Keegan, G. T. A. Huysmans, and E. Schwarz, Plasma Phys. Controlled Fusion 34(8), 1397 (1992).

${ }^{14}$ G. J. Kramer, S. E. Sharapov, R. Nazikian, N. N. Gorelenkov, and R. V. Budny, Phys. Rev. Lett. 92(1), 015001 (2004).

${ }^{15}$ S. Bernabei, M. G. Bell, R. V. Budny, E. D. Fredricksson, N. N. Gorelenkov, J. C. Hosea, R. Majeski, E. Mazzucato, C. K. Phillips, G. Schilling, and J. R. Wilson, Phys. Rev. Lett. 84(6), 1212 (2000).
${ }^{16}$ W. W. Heidbrink, E. D. Fredricksson, T. K. Mau, C. C. Petty, R. I. Pinsker, M. Porkolab, and B. W. Rice, Nucl. Fusion 39, 1369 (1999).

${ }^{17}$ P. Sandquist, S. E. Sharapov, M. Lisak, and T. Johnson, Phys. Plasmas 14, 122506 (2007).

${ }^{18}$ T. Gassner, K. Schoepf, S. E. Sharapov, V. G. Kiptily, S. D. Pinches, C. Hellesen, J. Eriksson, and JET-EFDA Contributors, Phys. Plasmas 19, 032115 (2012).

${ }^{19}$ E. M. Edlund, M. Porkolab, G. J. Kramer, L. Lin, Y. Lin, and S. J. Wukitch, Phys. Rev. Lett. 102, 165003 (2009).

${ }^{20}$ E. Joffrin, A. C. C. Sips, J. F. Artaud, A. Becoulet, L. Bertalot, R. Budny, P. Buratti, P. Belo, C. D. Challis, F. Crisanti, M. de Baar, P. de Vries, C. Gormezano, C. Giroud, O. Gruber, G. T. A. Huysmans, F. Imbeaux, A. Isayama, X. Litaudon, P. J. Lomas, D. C. McDonald, Y. S. Na, S. D. Pinches, A. Staebler, T. Tala, A. Tuccillo, K.-D. Zastrow, and JET-EFDA Contributors, Nucl. Fusion 45, 626 (2005).

${ }^{21}$ Y.-K. M. Peng and D. J. Strickler, Nucl. Fusion 26, 769 (1986).

${ }^{22}$ J. Candy, B. N. Breizman, J. W. Van Dam, and T. Ozeki, Phys. Lett. A 215, 299 (1996).

${ }^{23}$ B. N. Breizman and S. E. Sharapov, Plasma Phys. Controlled Fusion 37, 1057 (1995).

${ }^{24}$ C. Uberoi, Phys. Fluids 15(9), 1673 (1972)

${ }^{25}$ M. N. Rosenbluth and P. H. Rutherford, Phys. Rev. Lett. 34(23), 1428 (1974).

${ }^{26}$ J. Candy and M. N. Rosenbluth, Phys. Plasmas 1(2), 356 (1994).

${ }^{27}$ H. L. Berk, R. R. Mett, and D. M. Lindberg, Phys. Fluids B 5(11), 3969 (1993).

${ }^{28}$ M. Abramowitz and I. A. Stegun, Handbook of Mathematical Functions with Formulas, Graphs and Mathematical Tables (Dover Publications, New York, 1972). 